\title{
The impact of a fixed-target experiment with LHC beam for astroparticle physics
}

\author{
Colin Baus, Ralph Engel, Anatoli Fedynitch, Uwe Krämer, Tanguy Pierog, Felix \\ Riehn, Ralf Ulrich* \\ Karlsruhe Institute of Technology, Karlsruhe, Germany \\ E-mail: ralf.ulrichakit.edu
}

\begin{abstract}
There are two main points, where the data from a fixed-target experiment with LHC beam will contribute unique information. Firstly, to better understand the inclusive flux of atmospheric neutrinos at very high, $\mathrm{PeV}$, energies. Secondly, to the apparent over-abundance of $\mathrm{GeV}$ muons in ultra-high energy extensive air showers. To contribute towards answering these questions, the experimental limitations and requirements for a fixed-target experiment at LHC are presented and discussed. The investigation of forward D-meson production at high- $x_{\mathrm{F}}$ is essential in order to distinguish if $\mathrm{PeV}$ neutrinos are indeed astrophysical or may also be produced partly within the atmosphere. Furthermore, the production of $\mathrm{GeV}$ muons is deeply related to the pion cascade within air showers, and the corresponding pion-air interactions. More precise fixed-target data for pion-Carbon at LHC beam energies will contribute significantly to a better modeling of the muon content of air showers.
\end{abstract}

The 34th International Cosmic Ray Conference,

30 July- 6 August, 2015

The Hague, The Netherlands

* Speaker. 


\section{Introduction}

Many measurements performed in astroparticle physics depend in their interpretation on the precise knowledge of secondary particle production in hadronic collisions. Hadrons, for example, originating from the cosmic ray population, interact with nuclei and produce high energy photons, neutrinos, anti-particles, and extensive secondary particle cascades. In this context there are two main problems: often it is needed to extend the modeling of hadronic interactions much beyond the reach of man-made accelerators in center-of-mass energy and, furthermore, the most relevant particle production in terms of energy transport is directed towards very forward angles, making precise accelerator measurements very difficult.

Secondary particles carrying a large momentum fraction with respect to the initial projectile, typically expressed in terms of Feynman- $x$

$$
x_{\mathrm{F}}=\frac{p_{\|}}{p_{\max }} \approx \frac{2 p_{\|}}{\sqrt{s}}
$$

are the most relevant to understand the dissipation of the primary energy and the production of high energy secondary particles. The interaction of ultra-high cosmic ray primaries in the atmosphere leads to the formation of huge particle cascades, of which specific properties can be measured very reliably with state of the art experiments like the Pierre Auger Observatory [1] or Telescope Array [2]. At the same time, cosmic ray interaction in the atmosphere and secondary particle cascades produce an inclusive flux of atmospheric muons and neutrinos directed towards Earth [3]. These atmospheric leptons are an important background for, for example, the measurement of astrophysical neutrinos with the IceCube experiment [4]. But also in space the interaction of cosmic ray particles with matter leads to conventionally produced gamma-ray emission, and fluxes of positrons and anti-protons that can be measured at Earth. Such gamma-rays are known as the diffuse galactic gamma-ray flux observed, for example, with the Fermi satellite [5]. And the local flux of antiparticle is, of course, of central importance for dark matter searches.

Here we outline the relation between experimental acceptance and the particle production phase space relevant for specific important observations. It is argued that a fixed target experiment with LHC beam energy has the potential to extend the experimental coverage into the phase space of high $x_{\mathrm{F}}$, where no data exits at high energies. Contrary to LHC collider experiments, a fixed target experiment is much more flexible in the choice of the target material, such that measurements can be performed with nuclear systems where they are actually needed for.

The measurements of a dedicated fixed-target experiment at LHC are essential to support and complement the progress made in observational astroparticle physics in recent years. Precise understanding of hadronic and nuclear reactions in the relevant phase space has the potential to unveil the nature and sources of the highest energy particles in our local universe, both, charged as well as neutrinos. This is a fundamental step in exploring the highest-energy universe, also in the quest for new and unexpected physics.

There are currently two main critical questions in the field of cosmic-ray related particle production in the atmosphere. The first one is related to the observation of astrophysical PeV neutrinos [6] and the evaluation of the unknown background due to the charm content of hadrons. The second problem is the deficit of muons in the $\mathrm{GeV}$ range in extensive air shower of $\sim 10^{19} \mathrm{eV}$ [7]. 
Both issues are very closely linked to particle physics and the elementary QCD particle production mechanisms in the air shower cascades. With the existing collider experiments the phase-space coverage is not large enough to address these problems. On the other hand, fixed-target experiments with excellent phase-space coverage exist, for example, NA61 [8], but operate at beam energies below $\sim 350 \mathrm{GeV}$.

\section{Production of neutrinos and muons in air showers}

The inclusive flux of leptons in the atmosphere is primarily originating from semi-leptonic decays of light $\left(\pi, K^{ \pm}, K^{0}\right)$ and heavy mesons $\left(D^{ \pm}, D^{0}\right)$. The long decay time allows charged pions and kaons to travel significant distances through the atmosphere before decay. During the transport they can hadronically interact with air nuclei, where their kinetic energy is converted into new particles with a softer energy spectrum. For charmed mesons the decay length is more than four orders of magnitude shorter, thus, they decay instantly even at very high energies. The leptons from decays of charmed particles are therefore called prompt and the resulting leptons have an energy spectrum that resembles that of the incoming cosmic rays. The probability to produce charmed mesons is very small, but from the argument above, there must be a transition energy above which the prompt leptons will dominate the conventional lepton flux.

Within an approximate treatment of the cascade equations [3, 9] for inclusive secondary particle fluxes in air, the lepton fluxes are proportional to the corresponding spectrum weighted moments

$$
Z_{i j}=\int_{0}^{1} \mathrm{~d} x_{\text {lab }}\left(x_{\mathrm{lab}}\right)^{\gamma-1} \frac{\mathrm{d} n_{i+\mathrm{air} \rightarrow j+X}}{\mathrm{~d} x_{\text {lab }}},
$$

with $j=\pi^{ \pm}, K, D, \ldots$. Here the primary flux has been assumed to follow a power law $\Phi_{\mathrm{i}}(E) \propto$ $E^{-\gamma}$ with $i=\mathrm{p}, \mathrm{He}, \ldots$, Fe and the particle production cross sections have to satisfy approximately Feynman scaling. The energy fraction $x_{\text {lab }}=E_{j} / E_{i}$ of the secondary particles $j$ relative to that of the interacting particle $i$ is approximately equal to the Feynman- $x$ in forward direction.

Since particle production is weighted with $\left(x_{\mathrm{lab}}\right)^{\gamma-1}$ with $\gamma \approx 2.7$ the forward projectile fragmentation region is the most relevant. This is illustrated in Fig. 1. The acceptance of LHCb covers less than $10 \%$ of the relevant production phase space. Beyond that the cross-sections have to be extrapolated using models. This extrapolation is uncertain because of non-perturbative processes, which are not well understood, have to be taken into account in the phase-space where perturbative QCD calculations are not applicable.

Figure 2 shows the contributions of different $x_{\mathrm{F}}$-regions to the inclusive flux of muon neutrinos. Here we assume that particle production is known up to a lab. energy of $6.5 \mathrm{TeV}$ thanks to low-energy and LHC fixed-target experiments. The colored bands were calculated by integrating over all primary nucleon energies up to $6.5 \mathrm{TeV}$ and indicate what part of the lepton flux would be known unambiguously. Using the assumption of approximate Feynman scaling, the spectrum weighted moments are changing only slowly with collision energy. Hence, effectively, the theoretical uncertainties would be reduced up to even higher energies compared to what is shown, since the additional high-energy data-points from a fixed target experiment at LHC would notably constrain hadronic interaction models even at higher energy. 


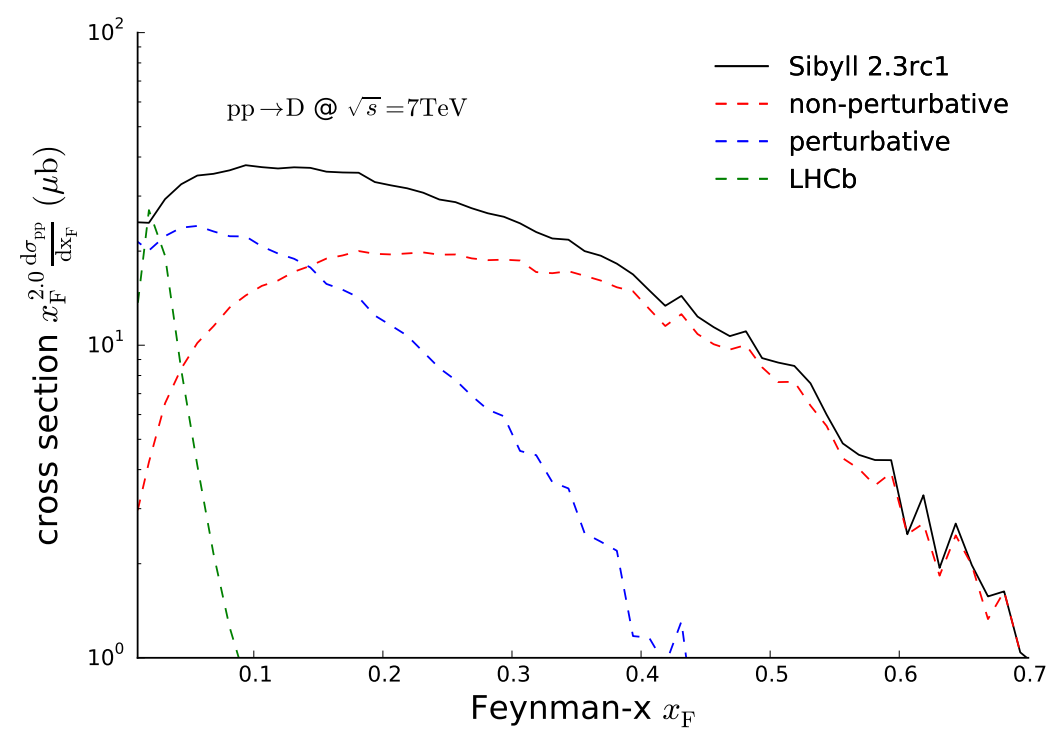

Figure 1: Production spectrum of $D$ mesons weighted by $\left(x_{\mathrm{lab}}\right)^{\gamma-1}$. The lines show the predictions of the Sib Yll $[10,11]$ model. Within this model there is a perturbative and a non-perturbative contribution to charm hadron production. The phase space covered by LHCb, the LHC experiment with the most forward reaching acceptance for charm particle identification, is also shown.

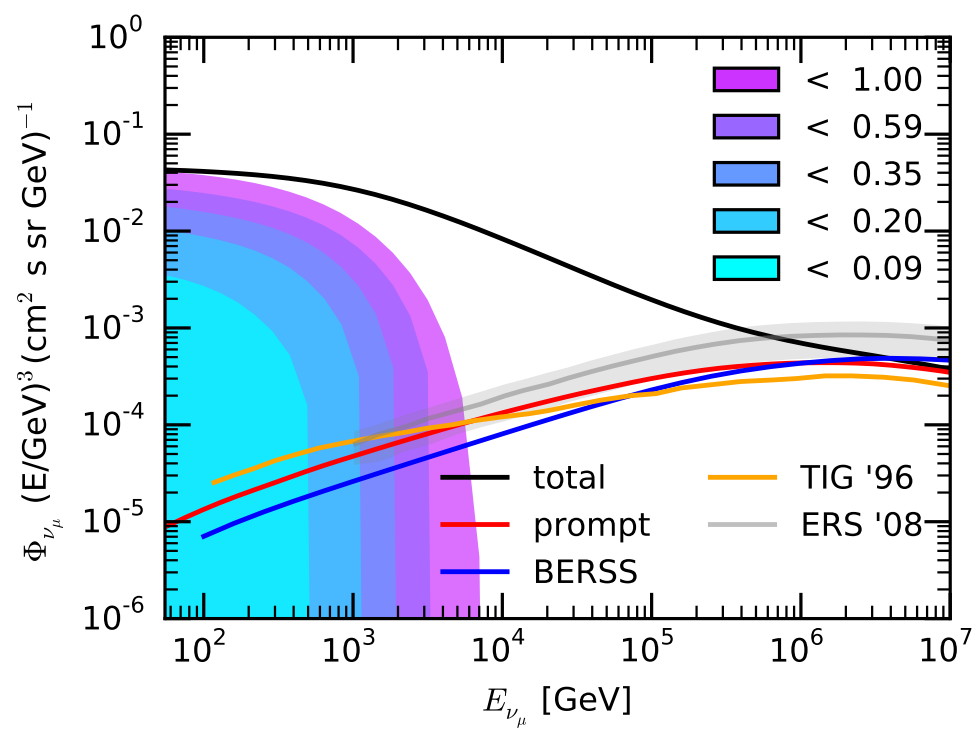

Figure 2: Inclusive flux of atmospheric muon neutrinos using a simple broken power-law primary spectrum from [12]. The colored bands illustrate the contribution of phase-space regions, cut on $x_{F} \approx x_{\text {lab }}$, with projectile energies up to $6.5 \mathrm{TeV}$. Conventional and prompt predictions are calculated using SIB YLL 2.3rc3 [10]. Other calculations of the prompt flux are BERSS [13], ERS [14] and TIG [12]. The calculation uses MCEQ [15], an efficient numerical solver for coupled cascade equations, and a new version of the SIBYLL interaction model $[10,11]$. 

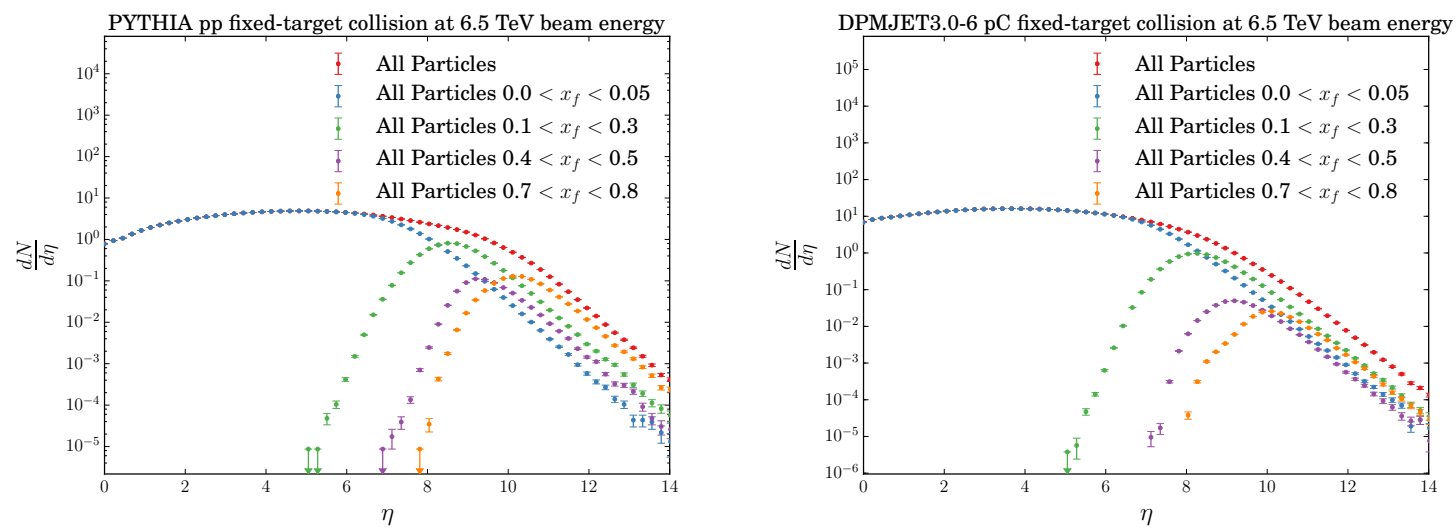

Figure 3: The ranges in $\eta$ where particles from specific $x_{\mathrm{F}}-$ intervals can be detected. Left panel: simulated with PYTHIA6 for pp collisions. Right panel: simulated with DPMJET-III for pC collisions.

A limit on the existence of an additional forward component in charmed quark production, such as intrinsic or spectator charm [16,17], will add more theoretical confidence in the reliability of results obtained within next-to-leading-order [13] or dipole model [14] calculations at small $p_{\perp}$ and large $x_{F}$. Reduced uncertainties on inclusive fluxes will, in turn, help to disentangle the ambiguities between the atmospheric and astrophysical neutrinos.

Finally we want to emphasize that a fixed-target experiment at $E_{\text {lab }}=6.5 \mathrm{TeV}$, corresponding to $\sqrt{s}=110 \mathrm{GeV}$, will provide data on forward hadron production in a particularly interesting energy range. The highest energies of current fixed-target measurements are $350-400 \mathrm{GeV}$ (CERN) and $800 \mathrm{GeV}$ (Tevatron, though only a few data sets exist with such a high beam energy). At these energies, hard interactions are very rare and have no influence on the bulk of the hadronic final states. These data sets are used extensively for tuning interaction models. But the dominant interaction processes at high energy are semi-hard interactions. Thus we face the problem that the forward physics aspects of the models are tuned on data without hard interactions, and later used in an energy domain that is dominated by hard interactions. Having a fixed target experiment that can bridge the gap between the two energy domains will be very useful and help to reduce the systematic uncertainties of the model predictions.

\section{Advantages and design considerations for a fixed-target experiment at LHC}

The LHC accelerator provides the currently by far highest energy controlled proton beam of currently $6.5 \mathrm{TeV}$ per proton. One of the main advantages of a fixed target setup is the very high variability of projectile-target combinations. With some preparation it is possible to extract primary protons and ions from the LHC beam, but also pions and kaons. The target can be chosen to be very close to typical scenarios in astroparticle physics, and one extremely well suited choice is carbon. It is the aim to study particle production in $\mathrm{pp}$, but in particular also for $\mathrm{pC}$ and also $\pi \mathrm{p}$ and $\pi \mathrm{C}$ collisions. Very little data exists on the relation between $\mathrm{pC}$ and $\pi \mathrm{C}$ collision at high energies. At the moment this is one of the uncertainties in the modeling of extensive air showers.

In order to make a meaningful measurement of spectrum-weighted moments that can yield new information on the overall normalisation as well as on the prompt component for the production of 

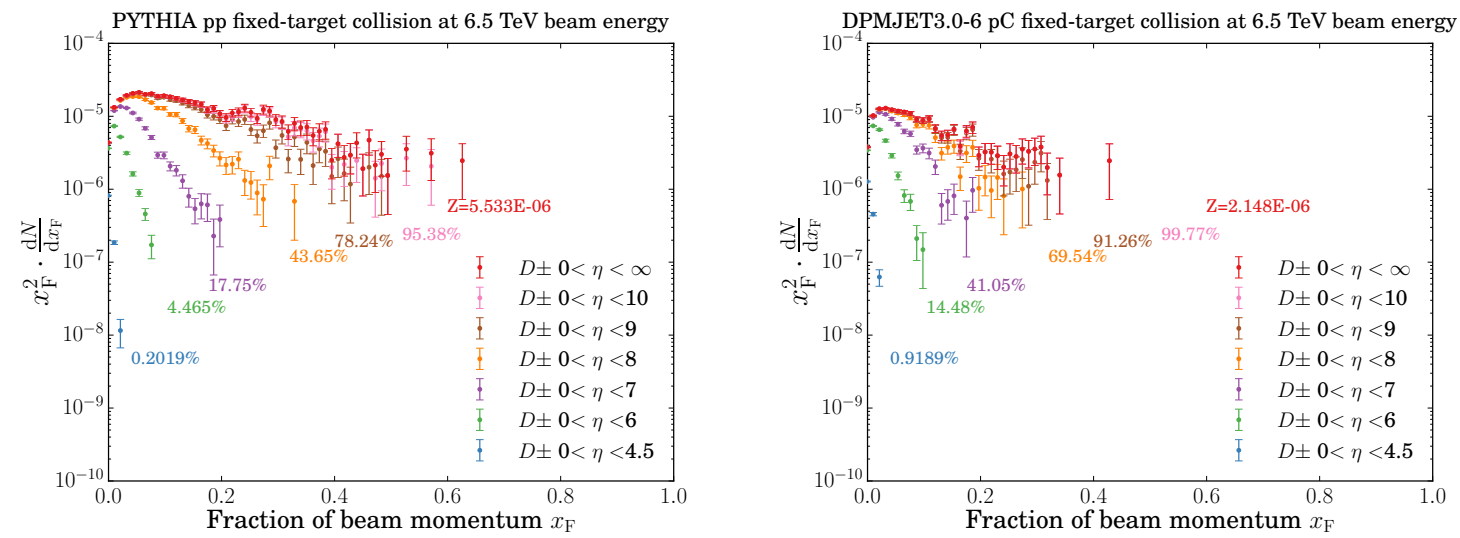

Figure 4: Acceptance with respect to the spectrum weighted moments of a fixed-target experiment at LHC with 6.5 TeV beam energy. Left panel: for pp collisions. Right panel: for $\mathrm{pC}$ collisions.

neutrinos and muons, the acceptance of the experiment must be at least up to $x_{\mathrm{F}} \sim 0.5$. In Fig. 3 the corresponding $\eta$ range for particles in specific $x_{\mathrm{F}}$ ranges are shown, both, for $\mathrm{pp}$ and $\mathrm{pC}$ fixed target collisions. This is equivalent to measure particle production up to $\eta=-\lg \tan \theta / 2 \sim 9$ and, thus, to forward angles $\theta$ down to about $2.5 \mathrm{mrad}$, which is essentially zero. In Fig. 4 it is shown for the example of spectrum weighted moments measured in $\mathrm{pp}$ and $\mathrm{pC}$ collisions, how important the reach in $\eta$ is to cover the phase space of the measurement. The acceptance of the LHCb experiment in $\eta$ up to 4.5 corresponds to less than one percent coverage of this essential measurement. It is highly non-trivial to measure secondary particle production using a $6.5 \mathrm{TeV}$ beam down to zero angle. For example, in order to precisely distinguish D-mesons, a vertex detector is needed close to the target area. Furthermore, particle identification must be provided to distinguish between pions,

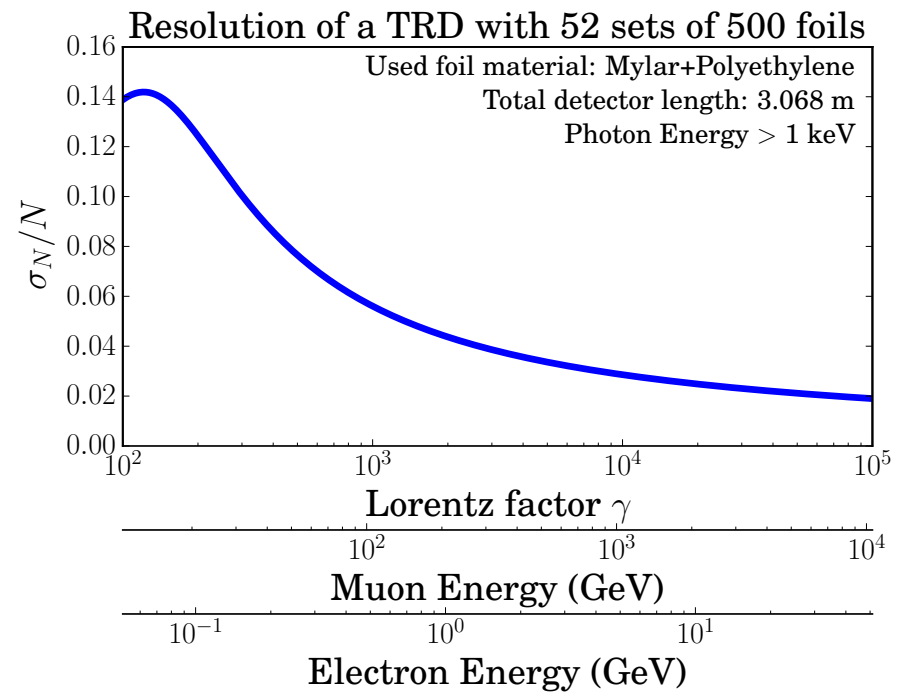

Figure 5: Resolution of a transition radiation tracker at high energies. Shown are the results as a function of $\gamma$, and also explicitly for muon and electrons as a function of the primary energy. 
muons and best electrons. This could be achieved with a multi-parameter approach by combining $\mathrm{d} E / \mathrm{d} X$, transition radiation, magnetic bending, time-of-flight, and calorimeter signature. Such a system can be optimized to require only the minimal magnetic bending power, which is the main cost-driving factor for any such experiment. In order to emphasize the need for a new type of experimental approach it is sufficient to calculate that in order to achieve a momentum resolution on the \%-level with a single-magnet bending system, an unrealistically high field on the order of $20 \mathrm{~T}$ on a length of $3 \mathrm{~m}$ is required. However, in particular for the highest energy secondaries, a comparable resolution can also be achieved with a transition radiation detector on a scale of $3 \mathrm{~m}$. See Fig. 5 (right). Such a transition radiation detector will have a material budget of $\approx 15 X_{0}$, and will thus act essentially as the first stage of an electromagnetic calorimeter with the possibility to see details of $\gamma$ conversions. A precise measurement of the muon $\gamma=E / m_{\mu}$ is equivalent to measure the muon energy, or momentum. At the same time, the simultaneous observation of the showering properties of the particles will discriminate efficiently between muons, electrons and pions/hadrons. This illustrates, how it is essential to overcome the strict separation in different separated particle detector concepts, and how an integrated particle flow detector must be designed from first principles. Dedicated studies about the capabilities of an optimized experimental layout are ongoing.

\section{Conclusion}

There are fundamental questions in astroparticle physics directly linked to a precise understanding of hadronic multi-particle production in the forward phase-space. The most prominent open problem at this moment is the question about the prompt charm contribution to the muon and neutrino production in extensive air showers. This will have major consequences on the interpretation of data from neutrino observatories, such as IceCube. A fixed-target experiment at $6.5 \mathrm{TeV}$ beam energy can exploit the existing LHC infrastructure in an optimal way to maximise the information gain for astroparticle physics. In this way also the investment in large next-generation neutrino and cosmic ray observatories will benefit significantly in scientific potential.

Already the design consideration for such a new fixed target experiment with a $6.5 \mathrm{TeV}$ primary beam are a major challenge. Space and resource optimization are a significant problem that require the development of a very integrated particle identification and reconstruction system.

\section{Acknowledgment}

This work is supported in part by the Helmholtz Young Investigator Group HHNG VH-NG-733 and the Helmholtz Alliance for Astroparticle Physics HAP, which are both funded by the Initiative and Networking Fund of the Helmholtz Association.

\section{References}

[1] Pierre Auger Collaboration, A. Aab et al., The Pierre Auger Cosmic Ray Observatory, to appear in Nucl. Instrum. Meth. A (2015) [1502.01323].

[2] Telescope Array Collaboration, T. Abu-Zayyad, R. Aida, M. Allen, R. Anderson, R. Azuma, et al., The surface detector array of the Telescope Array experiment, Nucl. Instrum. Meth. A689 (2012) 87-97, [1201.4964]. 
[3] T. K. Gaisser, Cosmic rays and particle physics. Cambridge Univ. Press,, Cambridge,, 1990.

[4] IceCube Collaboration, J. Ahrens et al., Sensitivity of the icecube detector to astrophysical sources of high energy muon neutrinos, Astropart. Phys. 20 (2004) 507-532, [a stro-ph/ 0305196 ].

[5] Fermi-LAT Collaboration, M. Ackermann et al., Fermi-LAT Observations of the Diffuse Gamma-Ray Emission: Implications for Cosmic Rays and the Interstellar Medium, Astrophys. J. 750 (2012) 3, [1202.4039].

[6] IceCube Collaboration, M. Aartsen et al., Observation of High-Energy Astrophysical Neutrinos in Three Years of IceCube Data, Phys. Rev. Lett. 113 (2014) 101101, [1405. 5303 ].

[7] Pierre Auger Collaboration, A. Aab et al., Muons in air showers at the Pierre Auger Observatory: Mean number in highly inclined events, Phys. Rev. D91 (2015), no. 3 032003, [1408.1421].

[8] NA61 Collaboration, N. Abgrall et al., NA61/SHINE facility at the CERN SPS: beams and detector system, JINST 9 (2014) P06005, [1401.4699].

[9] P. Lipari, TeV muons in hadronic showers, Astropart. Phys. 1 (1993) 399-416, [hep-ph/930 7289 ].

[10] F. Riehn, R. Engel, A. Fedynitch, T. K. Gaisser, and T. Stanev, A new version of the event generator Sibyll, Proc. of 34th Int. Cosmic Ray Conf., The Hague (2015).

[11] F. Riehn, R. Engel, A. Fedynitch, T. K. Gaisser, and T. Stanev, Charm production in SIBYLL, 1502.06353.

[12] P. Gondolo, G. Ingelman, and M. Thunman, Charm production and high-energy atmospheric muon and neutrino fluxes, Astropart. Phys. 5 (1996) 309-332, [hep-ph/ 9505417 ].

[13] A. Bhattacharya, R. Enberg, M. H. Reno, I. Sarcevic, and A. Stasto, Perturbative charm production and the prompt atmospheric neutrino flux in light of RHIC and LHC, 1502.01076.

[14] R. Enberg, M. H. Reno, and I. Sarcevic, Prompt neutrino fluxes from atmospheric charm, 0806.0418.

[15] A. Fedynitch, R. Engel, T. K. Gaisser, F. Riehn, and T. Stanev, Calculation of conventional and prompt lepton fluxes at very high energy, 1503.00544 . Proceedings of the International Symposium for Very High Energy Cosmic Ray Interactions (ISVHECRI 2014).

[16] S. J. Brodsky, P. Hoyer, C. Peterson, and N. Sakai, The Intrinsic Charm of the Proton, Phys. Lett. B93 (1980) 451-455.

[17] G. Ingelman and M. Thunman, Signals for intrinsic charm in high-energy interactions, Z. Phys. C73 (1997) 505-515, [hep-ph/9604289]. 